\title{
The Effect of Patellar Button Placement on Gait and Patellar Stresses Following Total Knee Arthroplasty
}

ISSN: 2576-8875

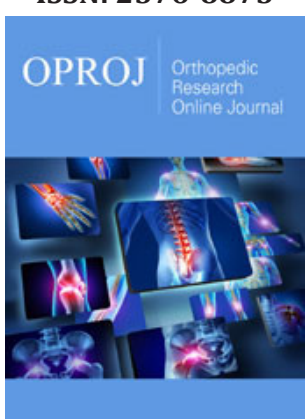

${ }^{* 1}$ Corresponding author: Dmitriy Peresada, College of Medicine, University of Illinois at Chicago, USA

Submission: 梅 November 01, 2019

Published: 留 November 08, 2019

Volume 6 - Issue 2

How to cite this article: Farid Amirouche, Giovanni Francesco Solitro, Dmitriy Peresada, Wayne M Goldstein, Mark H Gonzalez. The Effect of Patellar Button Placement on Gait and Patellar Stresses Following Total Knee Arthroplasty. Ortho Res Online J. 6(2). OPROJ.000631.2019. DOI: 10.31031/OPROJ.2019.06.000631

Copyright@: Dmitriy Peresada, This article is distributed under the terms of the Creative Commons Attribution 4.0 International License, which permits unrestricted use and redistribution provided that the original author and source are credited. Goldstein ${ }^{1}$ and Mark H Gonzalez ${ }^{1}$

${ }^{1}$ Department of Orthopaedic Surgery, University of Illinois at Chicago, USA

${ }^{2}$ College of Medicine, University of Illinois at Chicago, USA

\begin{abstract}
Background: In total knee arthroplasty with patellar resurfacing, surgeons are often faced with a choice of where to position the patellar button. Patellar button positioning has been studied in relation to patellar tracking and risk of subluxation. Consequently, medial placement has been recommended. However, postoperative gait and risk of periprosthetic complications have not been thoroughly addressed in relation to patellar button positioning.
\end{abstract}

Objective: To establish a generalized criterion for patellar button positioning during total knee replacement (TKA) to reduce the risk of postoperative gait abnormalities, patellar fracture, pain, and stress wear.

Methods: Five distinct button positions were analyzed. A previously-validated gait model was customized with data obtained from cadaveric patellae to evaluate individual quadriceps forces. The maximal combined quadriceps force during gait was applied to a finite element analysis model to calculate patellar von Mises stresses for all button positions, as well as for a large diameter button covering most of the patellar resurfaced area.

Results: Significant differences were found in average forces exercised by individual quadriceps muscles during gait. Distal and medial configurations produced the lowest individual quadriceps muscle forces. Bone stress ranged from 12.45MPa to $12.57 \mathrm{MPa}$. The button stress ranged from 11.27MPa to 17.20 MPa, with distal and centered placement, respectively.

Conclusion: Patellar button positioning influences muscle forces during gait and affects the stress on the patellar button itself. Distal and medial button positions are more likely to optimize postoperative gait kinematics, while the distal position may reduce button wear. These findings provide further insight into patellar button placement..

Keywords: Total knee arthroplasty; Knee replacement; Patellar button; Patellar resurfacing; Gait

Abbreviations: TKA: Total Knee Arthroplasty; UHMWPE: Ultra High Molecular Weight Polyethylene; CT: Computerized Tomography; [\%BW]: Percent Body Weight; [deg]: Degrees

\section{Introduction}

The annual demand for total knee arthroplasty (TKA) in the United States is projected to reach 3.48 million procedures by 2030 [1]. Of these, patellar resurfacing is indicated for patients with anterior knee pain, old age, rheumatoid arthritis, and advanced osteoarthritis [2]. In the US, more than $80 \%$ of primary TKAs include patellar resurfacing [3]. This procedure involves resecting of the articular surface of the patellar bone and securing a dome-shaped patellar button to the articular surface [2].

The efficacy and indications of patellar resurfacing remain disputed. Meta-analyses found that resurfacing reduced the risk of reoperation, without significantly affecting post-operative knee pain and knee scores [3,4]. The rate of patellofemoral complications for primary TKA is generally under $10 \%$ for both resurfaced [4,5] and non-resurfaced knees $[4,6,7]$, but some authors attribute an increase in complications to resurfacing. Such complications include pain [8], maltracking, instability, excessive thickness [6], button loosening [5], periprosthetic patellar fractures [9], and polyethylene wear [10].

To reduce such complications, current practice is to recreate the original thickness of the native patella [2]. If at least $12-15 \mathrm{~mm}$ of the residual patella cannot remain after resection, the 
procedure is usually not performed due to increased risk of failure of the residual bone [11]. Previous studies also determined that an oblique cut improves component durability [12]. Positioning of the patellar button provides another opportunity to reduce complications. Multiple studies that addressed risk of subluxation support medial positioning [2], because it better reproduces the anatomy of the native patella and improves patellar tracking $[11,13]$. There is also biomechanical evidence against distal button placement to avoid overloading the patella [14]. While authors have addressed the influence of patellar button position on patellofemoral contact forces $[14,15]$, the consequences of those forces on muscle forces during gait and on patellar stresses have yet to be investigated. A 3-to-9 fold increase in stress in the implanted patella was reported [16] and high patellofemoral stress was associated with increased risk of patellar fracture [9] and pain [8]. Additionally, there is a lack of guidelines on button positioning in relation to post-surgical gait.

The present study aimed to establish generalized criteria for patellar button positioning at time of TKA to reduce the risk of postoperative gait abnormalities, patellar fracture, pain, and component wear. We hypothesized that button positioning affects muscle forces during gait, as well as contact stresses on the button and on residual bone. We additionally evaluated the effects of button size to address conflicting findings in the literature $[7,17]$.

\section{Materials and Methods}

\section{Patellar button positions}

Gait and stress analysis were performed using a model of a single knee randomly selected from a group of cadaveric specimens. According to surgical technique, resurfacing specifications for the intact patella (a) were a cut of $10 \mathrm{~mm}$ compensated with an ultrahigh molecular weight polyethylene (UHMWPE) button of $10 \mathrm{~mm}$ thickness, $28 \mathrm{~mm}$ in diameter, and with curvature of $20 \mathrm{~mm}$. Five distinct patellar button positions were analyzed. The button was positioned at a point coinciding with the center of mass of the cut patella to define the centered position (b) and was repositioned by $5 \mathrm{~mm}$ in four directions from the centered position to define four additional cases: medial (c), lateral (d), proximal (e), and distal (f) (Figure 1). Repositioning the button from the centered position caused the overall center of gravity of the implanted patella to shift to in the corresponding direction (Table 1).

Table 1: Material properties of the cadaveric patella specimens before resurfacing and with the patella button in the centered position, as well as with the button shifted in four directions. Inertias are calculated in $\mathrm{x}, \mathrm{y}, \mathrm{z}$, and intermediate axes.

\begin{tabular}{|c|c|c|c|c|c|c|c|c|}
\hline & & \multicolumn{2}{|c|}{ Native Patella } & \multicolumn{5}{|c|}{ Patella with UHMWPE Button } \\
\hline & & $\begin{array}{l}\text { Arnold et al. } \\
\text { [19] }\end{array}$ & $\begin{array}{l}\text { CT based } \\
\text { characteriza- } \\
\text { tion }\end{array}$ & Centered & Proximal & Distal & Lateral & Medial \\
\hline \multicolumn{2}{|c|}{ Mass [g] } & 86.2 & 86.2 & \multicolumn{5}{|c|}{73.3} \\
\hline \multirow{3}{*}{\multicolumn{2}{|c|}{ Center of Mass Position [mm] }} & 1.8 & 1.8 & 2.7041 & 2.7041 & 2.7041 & 2.7041 & 2.7041 \\
\hline & & 26.4 & 26.442 & 26.442 & 27.392 & 25.492 & 26.442 & 26.442 \\
\hline & & 0 & 0 & 1.0707 & 1.0707 & 1.0707 & 1.0572 & -0.84306 \\
\hline \multirow{6}{*}{ Inertias [ $\left.\mathrm{g}^{*} \mathrm{~mm} 2\right]$} & $\operatorname{Ixx}$ & 2870 & 19920 & 15880 & 16170 & 16170 & 16150 & 16180 \\
\hline & Iyy & 13110 & 13390 & 11160 & 11160 & 11160 & 11430 & 11460 \\
\hline & Izz & 13110 & 11360 & 8612 & 8894 & 8894 & 8612 & 8612 \\
\hline & Ixy & 0 & -190 & -159.9 & 387.7 & 387.7 & -159.9 & -159.9 \\
\hline & $\mathrm{Ixz}$ & 0 & 26.73 & 175.4 & 175.4 & 175.4 & 722.9 & -372.2 \\
\hline & Iyz & 0 & 308.7 & -23.6 & -16.14 & -16.14 & -23.6 & -23.6 \\
\hline
\end{tabular}

\section{Muscle force evaluation}

Estimation of patellar cadaveric characteristics: To determine the post-surgery patellar kinematics, knee motion during extension was recorded with an Optotrak Certus $®$ motion capture system on three cadaveric specimens with a mean age of $56.4 \pm 10.8$ years. For the implantation, a sigma knee system CR150 with a round three-post patellar component and curved polyethylene (De Puy Orthopaedics, Warsaw, IN) was used. Femur, tibia, and patella were tracked in their six degrees of freedom using markers with an accuracy of $0.1 \mathrm{~mm}$. After digitalization of the anatomical axes, the patellar flexion and tilt angles were recorded with reference to the knee flexion angle from an unloaded position at 60 degrees of flexion to full extension. The load was imposed on the quadriceps tendon using a fiberwire suture passing through a pulley system mounted on a stable frame to control the force vector producing the flexion-extension, in accordance with previous similar experiments [18] (Figure 1). 

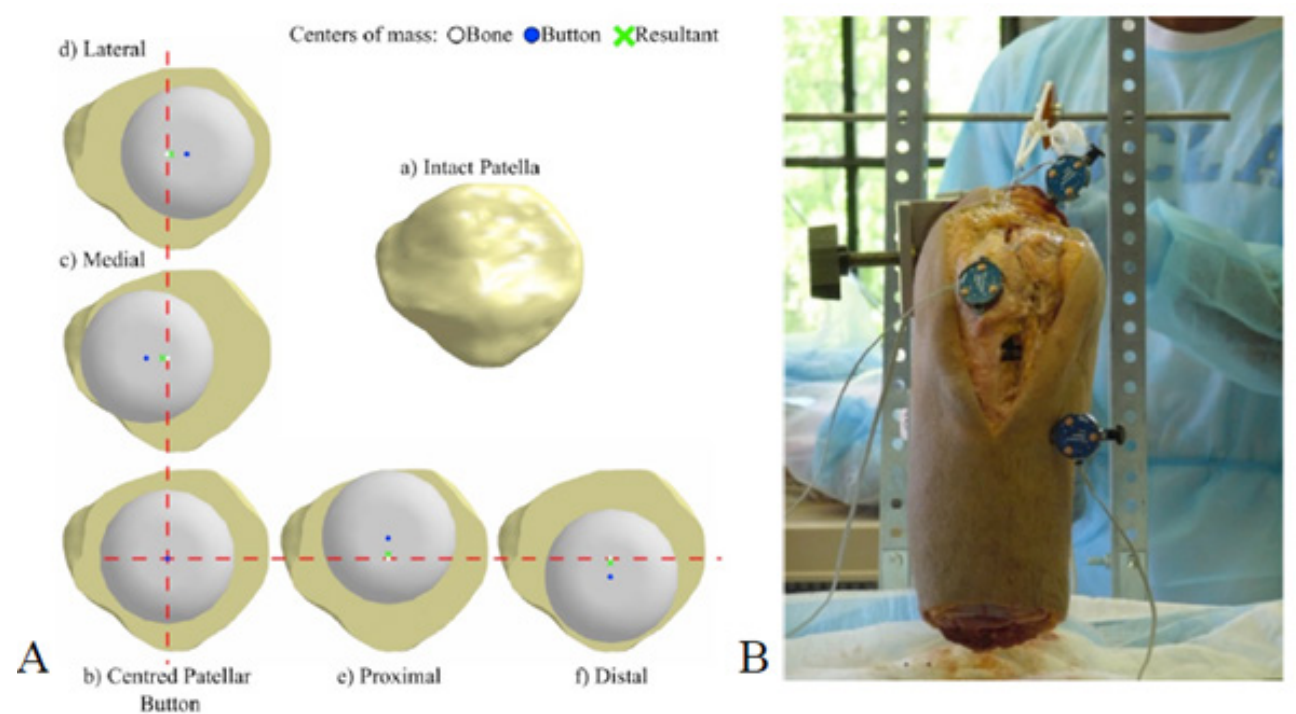

Figure 1: (A) Patellar button configurations considered in the Opensim gait model. The intact patella (a) and 5 variations in button placement (b-f) are shown. Centers of mass of the patellar bone, the patellar button, and the overall construct are indicated for each case. (B) Cadaveric knee specimen, with TKA implant and motion capture system in place

For the selected knee, 3D reconstruction of the patellar bone from CT images was performed using a Bright Speed (GE Healthcare Technologies, Waukesha, WI, USA) scanner (slice thickness of $0.625 \mathrm{~mm}$, pixel size of $0.422 \mathrm{~mm}$, and field view of $216 \mathrm{~mm}$ ) and the segmentation tools of the Mimics Suite (Materialise, Leuven, Belgium). Images of the entire knee were taken, without any surgical alterations, to develop a 3D reconstruction unaltered by the presence of the button. Mass properties for this set of CT images were accurately calculated using local bone mineral density. For the selected patella, mass properties in consequence of the 5 variation in button position were calculated by repositioning the patellar button (Table 1).

\section{Gait analysis}

To confer subject-specific realism, a previously-validated Opensim gait model [19] was customized for the cadaveric knee. The 3D reconstruction of the patellar bone from CT images of the cadaveric knee and the measured patellar tilt, flexion, and material properties (Table 1) were used to overwrite the patellar joint constants. Considering previous findings showing that gait kinematics are not influenced by TKA $[20,21]$, kinematic data was kept the same for all five button configurations. To evaluate the susceptibility of the TKA construct to complications in the form of gait abnormalities, muscle forces corresponding to the variations in button position were calculated using the built-in static optimization tool [22].

\section{Stress analysis}

Button position: Following the identification of muscle forces, we performed a stress analysis on the implanted patella during gait, considering the patellofemoral and tibiofemoral joints and a friction coefficient of 0.01 [23] at both interfaces (Figure 2). An UHMWPE button that was rigidly connected to the patellar bone via a flat surface was used in the model. The contact between the button and the condyles was solved with the augmented Lagrange method. The femur bone was assembled as a fixed component in the mediolateral and proximodistal directions, while it had free motion in the anteroposterior direction, in reference to the patella. The tibia was assembled as a fixed geometric component. The patella was fixed in the mediolateral and anteroposterior directions and had free motion in the proximodistal direction. The relative locations of the bony segments were extracted from CT images. To simulate the physiological loading of the patella at peak loads, muscle tensile forces were applied along the directions of the quadriceps tendons. A total quadriceps muscle force of $1127.76 \mathrm{~N}$ was applied to the patellar tendon in the unflexed knee position. Susceptibility of the implanted patella to failure modes such as fracture, pain, and component wear was evaluated by obtaining values of von Mises stress using a built-in functionality of ANSYS for the solution of the contact (ANSYS, Canonsburg, PA) for the five button positions (Figure 1).

Button sizing: To identify the benefits of a larger button, we made the button diameter such that it maximized the coverage of the resected patellar surface, while remaining entirely on the surface. The edge of the button coincided with three points on the edge of the resected bone: a superomedial point (A), near the insertion of the vastus medialis portion of the quadriceps tendon; a superolateral point (B), near the insertion of the vastus lateralis portion of the quadriceps tendon; and an inferolateral point (C), near the origin of the patellar tendon. The edge of the button was in proximity of an inferomedial point (D), near the origin of the patellar tendon. This resulted in a $35.17 \mathrm{~mm}$ button diameter, compared to the standard $28 \mathrm{~mm}$. The von Mises stresses were determined for this maximal button diameter using the same simulation conditions that were disclosed for the variation in button position. 


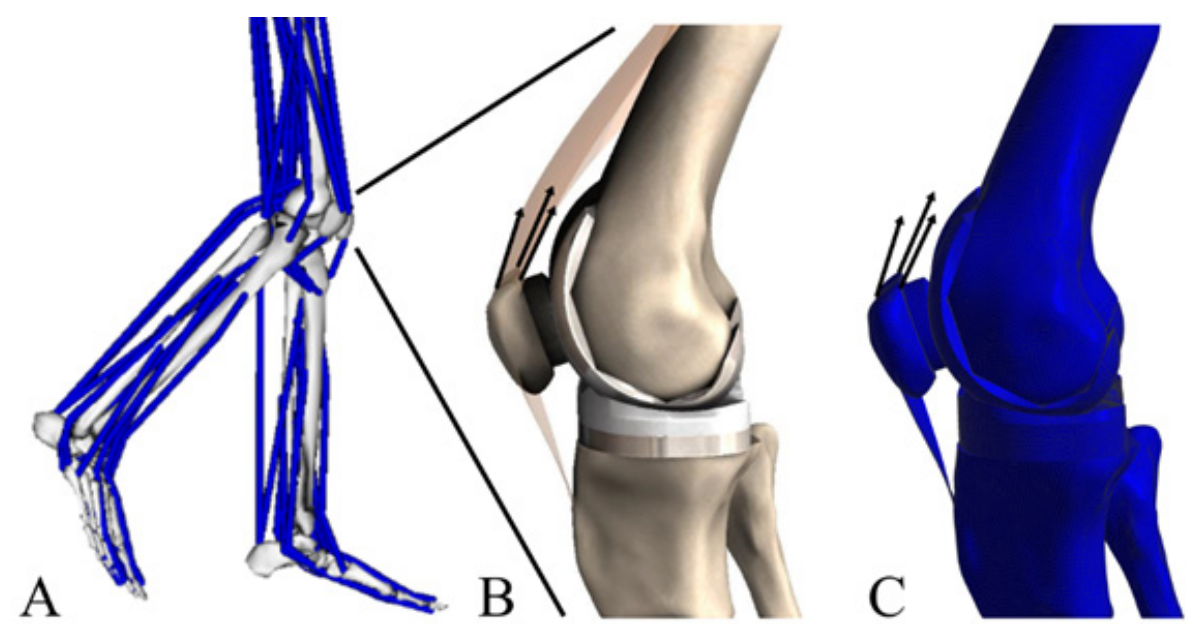

Figure 2: Expanded 3-D model of the knee joint used in stress analysis.

\section{Results and Discussion}

\section{Muscle force evaluation}

Cadaveric patellar characteristics: Kinematic analysis performed on post-TKA cadaveric knee specimens with the button in the central position resulted in plots of patellar extension and tilt versus knee flexion. The maximal patellar flexion of $35.81 \pm 2.72$ degrees was achieved at 60 degrees of knee flexion. Patellar tilt increased with knee flexion, until reaching a maximum of $1.57 \pm 0.76$ degrees, and then decreased (Figure 3).
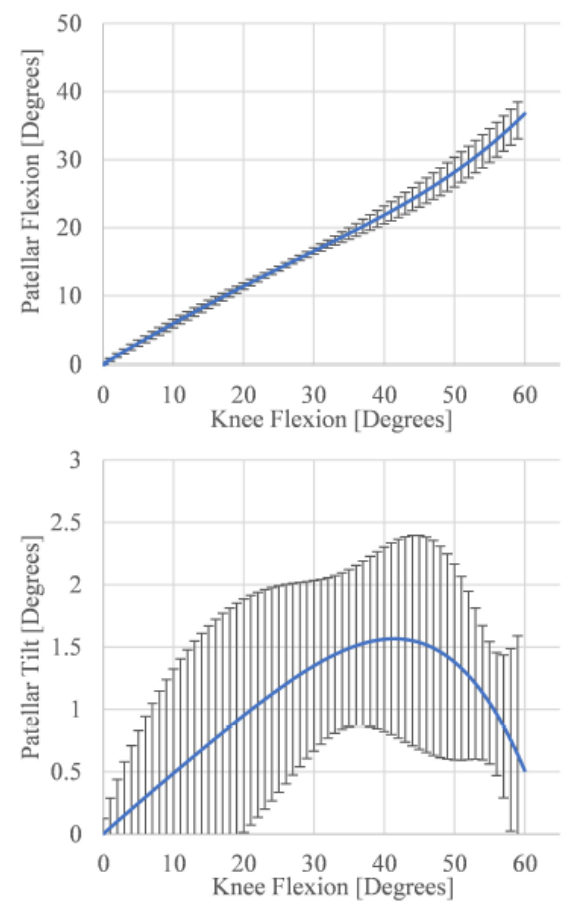

Figure 3: Patellar flexion and lateral tilt recorded from cadaveric knee specimens after TKA implants with the patellar button in the central position. Standard deviation is shown. This data was used to customize the Opensim model of gait analysis.

Individual muscle loads: The greatest differences in peak forces of individual muscles between various button positions were on the order of 4.8-18.3 percent bodyweight. The distal position resulted in lowest peak forces for the rectus femoris, vastus intermedialis, and vastus medialis. The medial position produced the lowest peak force for the vastus lateralis and second lowest peak forces for the other three quadriceps muscles (less than $1.2 \%$ bodyweight greater than the distal position). For each of the analyzed muscles, except for the rectus femoris, the centered position resulted in the highest peak force. In terms of the average forces exercised by the four quadriceps muscles, no statistically significant differences were found in relation to the button position using the Wilcoxon signed-rank test, with p-values greater than 0.6 and as high as 0.9531 (Table 2). 
Table 2: Gait analysis as demonstrated by forces, in percent of body weight [\%BW], produced by the quadriceps muscles. No significant differences were found, in terms of average forces produces by each muscle, between the different positions of the patellar button. The distal position resulted in the lowest maximal forces in three of the four muscles analyzed.

\begin{tabular}{|c|c|c|c|c|}
\hline \multicolumn{5}{|c|}{ Forces $[\% \mathrm{BW}]$} \\
\hline Rectus Femoris & Average & SD & Max & p-value \\
\hline Centered & 15.9 & 24.8 & 117.0 & -- \\
\hline Medial & 16.1 & 25.1 & 113.1 & 0.8557 \\
\hline Lateral & 16.7 & 26.3 & 114.1 & 0.7244 \\
\hline Proximal & 16.3 & 26.4 & 118.2 & 0.7465 \\
\hline Distal & 17.1 & 27.7 & 112.2 & 0.6278 \\
\hline \multicolumn{5}{|c|}{ Vastus Intermedialis } \\
\hline Centered & 4.6 & 8.1 & 34.1 & -- \\
\hline Medial & 4.6 & 7.7 & 27.7 & 0.6330 \\
\hline Lateral & 4.5 & 8.2 & 32.0 & 0.9531 \\
\hline Proximal & 4.4 & 8.0 & 32.8 & 0.8890 \\
\hline Distal & 3.9 & 7.2 & 26.5 & 0.6527 \\
\hline \multicolumn{5}{|l|}{ Vastus Lateralis } \\
\hline Centered & 14.6 & 29.1 & 123.6 & -- \\
\hline Medial & 14.9 & 28.8 & 105.3 & 0.6396 \\
\hline Lateral & 14.2 & 28.2 & 108.7 & 0.9458 \\
\hline Proximal & 14.1 & 28.3 & 106.0 & 0.8313 \\
\hline Distal & 14.4 & 29.0 & 111.3 & 0.7382 \\
\hline \multicolumn{5}{|l|}{ Vastus Medialis } \\
\hline Centered & 6.2 & 11.1 & 43.4 & -- \\
\hline Medial & 6.1 & 10.5 & 37.7 & 0.6356 \\
\hline Lateral & 6.0 & 11.0 & 41.5 & 0.9458 \\
\hline Proximal & 5.9 & 10.8 & 40.6 & 0.8673 \\
\hline Distal & 5.2 & 9.9 & 36.7 & 0.6848 \\
\hline
\end{tabular}

Table 3: Peak quadriceps force values during gait, for the first and second stances. Forces expressed in percent of body weight [\%BW] and in Newtons [N]. Corresponding knee flexion angles are indicated in degrees [deg].

\begin{tabular}{|c|c|c|c|c|c|c|c|}
\hline & Knee Angle [deg] & Quadriceps & Rec. fem. & Vas.int. & Vas. lat. & Vas. med. & Units \\
\hline \multirow{3}{*}{ First Stance } & \multirow{3}{*}{9.7} & 152.0 & 13.6 & 22.4 & 85.2 & 30.8 & \multirow{4}{*}[\%\mathrm{BW}]{} \\
\hline & & 150.6 & 9.4 & 21.5 & 89.1 & 30.7 & \\
\hline & & 149.8 & 1.2 & 22.6 & 93.2 & 32.7 & \\
\hline \multirow[t]{2}{*}{ Averages } & & 150.8 & 8.0 & 22.2 & 89.2 & 31.4 & \\
\hline & & 1073.8 & 57.2 & 157.8 & 635.3 & 223.6 & {$[\mathrm{~N}]$} \\
\hline \multirow{3}{*}{ Second Stance } & \multirow{3}{*}{10.1} & 172.8 & 1.1 & 27.6 & 103.8 & 40.4 & \multirow{4}{*}[\%\mathrm{BW}]{} \\
\hline & & 183.3 & 14.7 & 19.8 & 114.5 & 34.4 & \\
\hline & & 159.7 & 3.2 & 24.0 & 97.7 & 34.8 & \\
\hline \multirow[t]{2}{*}{ Averages } & & 171.9 & 6.3 & 23.8 & 105.3 & 36.5 & \\
\hline & & 1224.6 & 45.0 & 169.3 & 750.2 & 260.2 & {$[\mathrm{~N}]$} \\
\hline
\end{tabular}



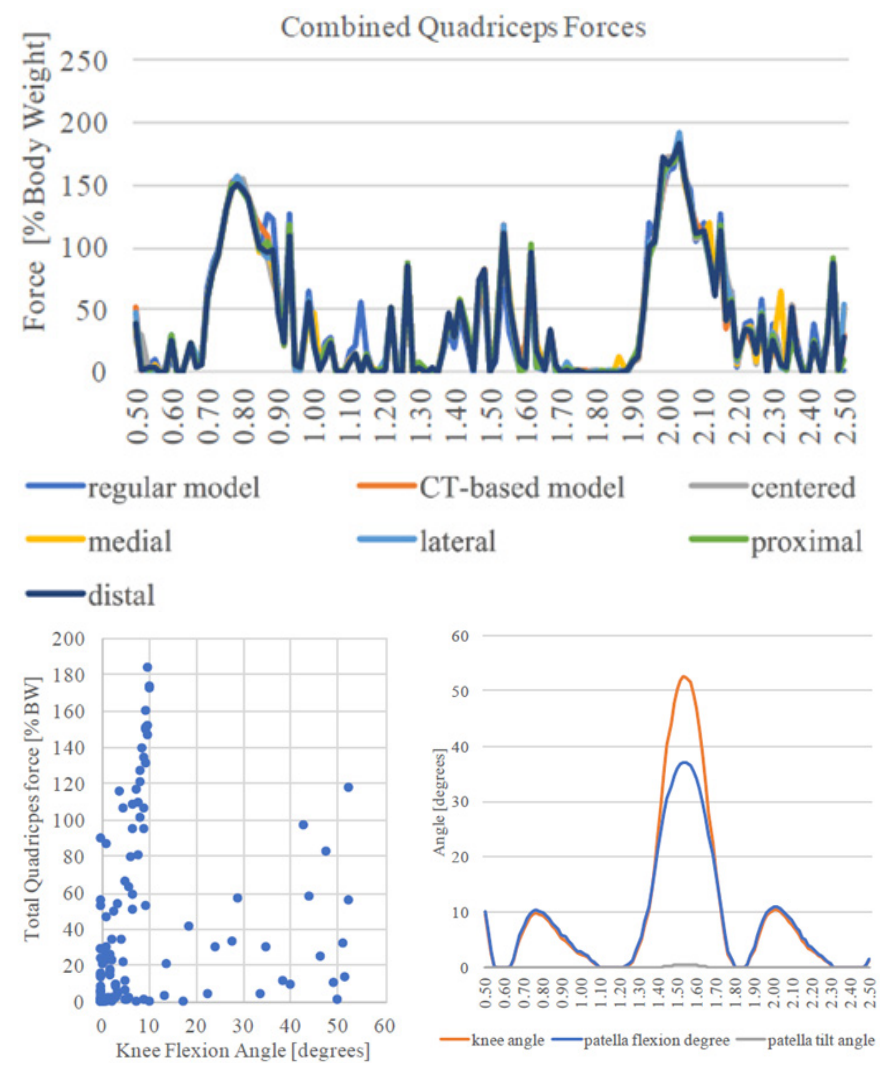

Figure 4: Gait analysis. (A) Combined quadriceps forces during the gait cycle. Plots for varying button configurations are superposed. Two stance positions are evidenced by peaks in forces. (B) Kinematics of the patellofemoral joint during the gait cycle. (C) Quadriceps loads, in percent body weight [\%BW], as function of knee flexion [degrees]. Peak loads occurred below 10 degrees of flexion.

For all the configurations, the resultant quadriceps force during gait had two peaks, representing the two stance phases. For a body weight of $72.6 \mathrm{Kg}$, in the CT-based pre-TKA configuration, this resulted in a total applied load of $1073.8 \mathrm{~N}$ at 9.7 degrees of knee flexion in the first stance and $1224.6 \mathrm{~N}$ at 10.1 degrees flexion in the second stance (Table 3) (Figure 4). During the entire gait cycle, most of the quadriceps loads were contained within 10 degrees of knee flexion and force higher than $120 \%$ of body weight were seen only below 10 degrees (Figure 4).

\section{Stress analysis}

Patellar component stress: At the residual patellar bone, stresses ranged from a minimum of $12.45 \mathrm{MPa}$ to a maximum of $12.57 \mathrm{MPa}$, with the proximal and distal configurations, respectively. The medial position produced a stress of $12.46 \mathrm{MPa}$ on the patellar bone. At the patellar button, stress ranged from a minimum of $11.27 \mathrm{MPa}$ to a maximum of 17.20MPa, with the distal and centered configuration, respectively (Figure 5).

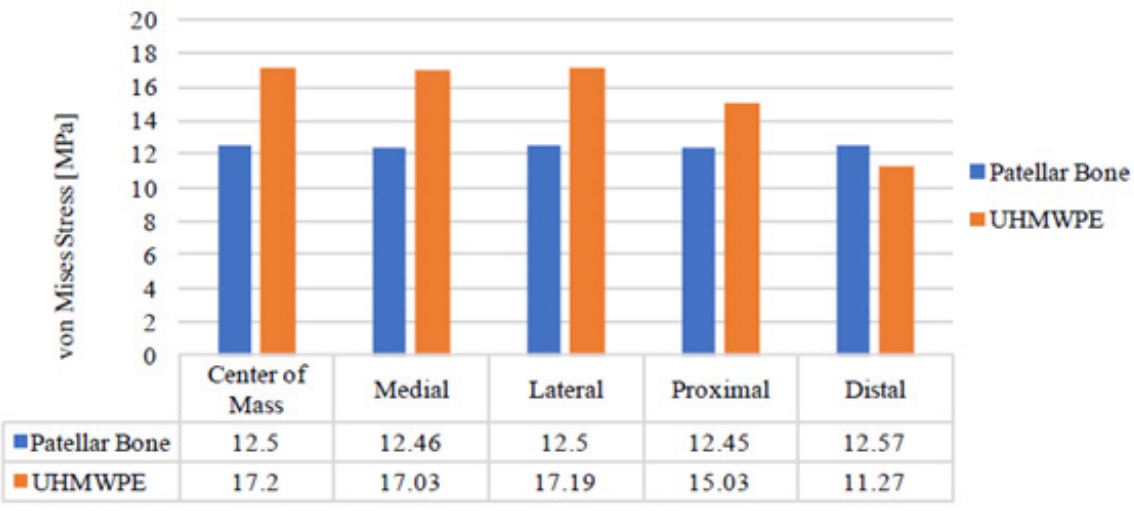

Figure 5: Distribution of von Mises stress, in MPa, on the residual patellar bone and on the UHMWPE button with various patellar button configurations. 
Influence of button size on stress: With the patellar button diameter maximized to $35.17 \mathrm{~mm}$, the stress at the residual patellar bone was $12.56 \mathrm{MPa}$, which was higher than the stress of $12.50 \mathrm{MPa}$ with the centered configuration. At the UHMWPE patellar button, stress with the maximal diameter was $12.09 \mathrm{MPa}$, which is lower than the stress of $17.20 \mathrm{MPa}$ with the centered configuration.

Discussion: Previous patellar button studies focused on mediolateral button positioning $[11,13]$ in relation to patellar tracking, usually without addressing gait. However, TKA was found to affect quadriceps activation during gait [24] and distally placed buttons were shown to increase the combined quadriceps force [14]. Considering that TKA appears to play a role in post-surgical joint kinetics, our study aimed to reduce the rate of fractures and gait abnormalities by optimizing the patellar button position with regard to these factors.

We found variability in peak gait forces between different button positions for individual quadriceps muscles. Distal placement produced the lowest peak forces for three of the four muscles. The medial position resulted in lowest peak force for the vastus lateralis and similar peak forces to the distal placement for the other three muscles. These results suggest that distal and medial positions are best at optimizing post-surgical gait kinetics, as evidenced by lower quadriceps forces. No studies are currently available for a direct comparison of our findings. Nakamura et al., found an increased combined maximum quadriceps force for a medio-distally placed button. They analyzed medialized button positioning in the proximal-distal direction, with $3 \mathrm{~mm}$ offsets from the center position. It must also be noted that they utilized a squatting motion, which is difficult for most TKA patients and for which peak loads are three times greater those we found for gait [14]. One limitation of our study is the use of the same kinematic data to model gait for all experimental conditions. However, previous studies showed no difference in postoperative kinematics between resurfaced and unresurfaced patellae $[20,21]$. We were also limited by the shapes of a Wiberg type II patella and a circular button.

Once we investigated how the patella is loaded during gait, we utilized these loads to perform a contact stress analysis on the resurfaced knee joint. Our calculations of stress on the resurfaced patella were consistent with the results of an earlier study, which found a maximum stress of 20MPa on the intact patella during gait [25]. Button positioning produced insignificant changes in stress on the residual patellar bone, with values of 12.45-12.57MPa. High patellofemoral stress is linked to bone fracture and pain $[8,9]$. Thus, within the limits of our experimental protocol, button position may not be an effective strategy to reduce these specific complications. We found significant differences in button stress. Distal placement resulted in button stress of $11.27 \mathrm{MPa}$, which is $25 \%$ lower than the second-lowest-stress (proximal) configuration. Yield values for UHMWPE (23MPa) [26] are above the maximal stresses on the button that we observed (17.20MPa). This is evidenced by the fact that catastrophic button wear usually occurs decades after the procedure $[5,6,10]$. However, differences in button stress during gait may become significant when extrapolated to a squatting motion, which involves greater patellofemoral forces [14]. Also, because we modeled a rigid connection between the button and the bone, we did not account for failure by loosening of the button or breaking of the pegs. However, these failure mechanisms are rare $[5,6]$.

Current literature reveals contradicting recommendations regarding size of the patellar button [7,15,17]. Atzori et al. [17] previously found superior clinical outcomes among patients with $38 \mathrm{~mm}$ and $41 \mathrm{~mm}$ buttons [17]. As an addition to the stress analysis, we maximized the button diameter to $35.17 \mathrm{~mm}$, according to the patellar dimensions, to monitor the benefits in terms of stress on the bone and on the button. We found a similar in stress on the bone (12.56MPa) compared to the regular $(28 \mathrm{~mm})$ sized button in the five analyzed positions. Stress on the button (12.09MPa) was the second-lowest, compared to the other configurations. Meding et al. [7] found a correlation between large ( $37 \mathrm{~mm})$ buttons and increased risk of patellar fracture in a large retrospective analysis [7]. Our model did not factor in an increase in button thickness that is associated with a larger button. Thicker button would require increased patellar resection, leading to higher periprosthetic patellar fracture risk.

\section{Conclusion}

In conclusion, patellar button positioning influences muscle forces during gait and affects the stress on the patellar button itself. Based on our analysis, distal and medial button positions are more likely to optimize postoperative gait kinematics. Distal positioning may also reduce the rate of button wear. Currently, there is overwhelming evidence in support of medialization of the patellar button to improve patellar tracking. Thus, when medial positioning is not possible based on patellar anatomy or prosthetic component dimensions, the distal position may be considered.

\section{Acknowledgement}

The work was partially supported by the Aurelio M. Caccomo Family Foundation.

\section{References}

1. Kurtz S, Ong K, Lau E (2007) Projections of primary and revision hip and knee arthroplasty in the United States from 2005 to 2030. J Bone Joint Surg Am 89(4): 780-785.

2. Abdel MP, Parratte S, Budhiparama NC (2014) The patella in total knee arthroplasty: To resurface or not is the question. Curr Rev Musculoskelet Med 7(2): 117-124.

3. Fraser JF, Spangehl MJ (2016) International rates of patellar resurfacing in primary total knee arthroplasty, 2004-2014. J Arthroplasty 32(1): 1-4.

4. He JY, Jiang LS, Dai LY (2011) Is patellar resurfacing superior than nonresurfacing in total knee arthroplasty? A meta-analysis of randomized trials. Knee 18(3): 137-144.

5. Mulhall KJ, Ghomrawi HM, Scully S (2006) Current etiologies and modes of failure in total knee arthroplasty revision. Clin Orthop Relat Res 446: 45-50.

6. Schiavone-Panni A, Cerciello S, Del Regno C (2014) Patellar resurfacing complications in total knee arthroplasty. Int Orthop 38(2): 313-317.

7. Meding JB, Fish MD, Berend ME (2008) Predicting patellar failure after total knee arthroplasty. Clin Orthop Relat Res 466(11): 2769-2774. 
8. Farrokhi S, Keyak JH, Powers CM (2011) Individuals with patellofemoral pain exhibit greater patellofemoral joint stress: a finite element analysis study. Osteoarthr Cartil 19(3): 287-294.

9. Chalidis BE, Tsiridis E, Tragas AA (2007) Management of periprosthetic patellar fractures. A systematic review of literature. Injury 38(6): 714724.

10. Lygre SHL, Espehaug B, Havelin LI (2011) Failure of total knee arthroplasty with or without patella resurfacing. Acta Orthop 82(3): 282-292.

11. Malo M, Vince KG (2003) The unstable patella after total knee arthroplasty: etiology, prevention, and management. J Am Acad Orthop Surg 11(5): 364-371.

12. Amirouche F, Choi KW, Goldstein WM (2013) Finite element analysis of resurfacing depth and obliquity on patella stress and stability in TKA. J Arthroplasty 28(6): 978-984.

13. Miller MC, Zhang AX, Petrella AJ (2001) The effect of component placement on knee kinetics after arthroplasty with an unconstrained prosthesis. J Orthop Res 19(4): 614-620.

14. Nakamura S, Tanaka Y, Kuriyama S (2017) Superior-inferior position of patellar component affects patellofemoral kinematics and contact forces in computer simulation. Clin Biomech 45: 19-24.

15. Fuchs S, Schütte G, Witte H, Rosenbaum D (2002) What influence do size and placement of patella resurfacing have on knee endoprosthesis? Unfallchirurg 105(1): 44-48.

16. Lee YS, Lee TQ Keyak JH (2009) Effect of an UHMWPE patellar component on stress fields in the patella: A finite element analysis. Knee Surgery, Sport Traumatol Arthrosc 17(1): 71-82.
17. Atzori F, Sabatini L, Deledda D (2015) Evaluation of anterior knee pain in a PS total knee arthroplasty: the role of patella-friendly femoral component and patellar size. Musculoskelet Surg 99(1): 75-83.

18. Sakai N, Luo ZP, Rand J (1996) Quadriceps forces and patellar motion in the anatomical model of the patellofemoral joint. Knee 3(1-2): 1-7.

19. Arnold EM, Ward SR, Lieber RL, Delp SL (2010) A model of the lower limb for analysis of human movement. Ann Biomed Eng 38(2): 269-279.

20. Pollo FE, Jackson RW, Koëter S (2000) Walking, chair rising, and stair climbing after total knee arthroplasty: patellar resurfacing versus nonresurfacing. Am J Knee Surg 13(2): 103-108.

21. Smith AJ, Lloyd DG, Wood DJ (2006) A kinematic and kinetic analysis of walking after total knee arthroplasty with and without patellar resurfacing. Clin Biomech 21(4): 379-386.

22. Delp SL, Anderson FC, Arnold AS (2007) Open Sim: Open-source software to create and analyze dynamic simulations of movement. IEEE Trans Biomed Eng 54(11): 1940-1950.

23. Ingrassia T, Nalbone L, Nigrelli V (2013) Finite element analysis of two total knee joint prostheses. Int J Interact Des Manuf 7(2): 91-101.

24. Li K, Ackland DC, McClelland JA (2013) Trunk muscle action compensates for reduced quadriceps force during walking after total knee arthroplasty. Gait Posture 38(1): 79-85.

25. Bischoff JE, Hertzler JS, Mason JJ (2009) Patellofemoral interactions in walking, stair ascent, and stair descent using a virtual patella model. J Biomech 42(11): 1678-1684.

26. Sobieraj MC, Rimnac CM (2009) Ulta high molecular weight polyethylene: Mechanics, morphology, and clincial behavior. J Mech Behav Bomed Mater 2(5): 433-443. 